

\title{
One-dimensional spinless fermion model with competing interactions beyond half-filling
}

\author{
A. K. Zhuravlev and M. I. Katsnelson
}

October 16, 2018

\begin{abstract}
An accurate numerical consideration of 1D spinless fermion model with next-nearest neighbour (NNN) interactions is carried out for the electron concentrations $4 / 7$. It is shown that depending on the parameters of the model it can be either Luttinger liquid or bipolaron liquid. In the former case competing interactions can result in a smooth behavior of one-electron distribution function at the Fermi surface with a divergence in the second derivative with respect to the quasimomentum (and not in the first one, as usual). In this connection, uncommon photoemission spectra for some 1D conductors can be explained qualitatively.
\end{abstract}

PACS 71.10.Pm, 71.10.Fd

Frustrations and competing interactions are very important for strongly correlated electron systems. As it was shown in a seminal work 1] they can change drastically a ground state of quantum antiferromagnets leading to the formation of "resonating valence bond" (RVB), or quantum spin liquid, state (see also 22] and references therein). This state has been found later in a series of compounds with both magnetic (spin liquid) and charge (pseudospin liquid) degrees of freedom, see, e.g., the discussion in Refs. [3, 使. The effects of competing interactions were investigated recently for one-dimensional (1D) spinless fermion model[5, 6, 7]. Usually this model is used for the description of Verwey metal-insulator transitions and charge ordering phenomena in $\mathrm{Fe}_{3} \mathrm{O}_{4}, \mathrm{Ti}_{4} \mathrm{O}_{7}$ and other d-metal compounds 8, 9, 10]. It is expected that in the half-filled case the growth of the Coulomb interaction leads to the transition from the metallic state to the insulating charge-ordered one. It appeared, however, that the competing interactions can result in the stabilization of metallic phase without charge ordering for arbitrarily strong Coulomb interaction [5, 6], this phase being not usual (for 1D systems) Luttinger liquid[7]. Here we investigate this model beyond half-filling.

We proceed with the Hamiltonian

$$
H=-t \sum_{i=1}^{L}\left(c_{i}^{\dagger} c_{i+1}+c_{i+1}^{\dagger} c_{i}\right)+V \sum_{i=1}^{L} n_{i} n_{i+1}+V^{\prime} \sum_{i=1}^{L} n_{i} n_{i+2}
$$

\footnotetext{
*Institute of Metal Physics, Ekaterinburg 620219, Russia
} 
where $c_{i}^{\dagger}, c_{i}$ are Fermi creation and annihilation operators on site $i, n_{i}=c_{i}^{\dagger} c_{i}$. Further we use periodic boundary conditions ( $L$ is the length of the ring) and put $t=1$ at the presentation of the computational results.

Earlier we investigated the cases $\rho=1 / 2$ and $\rho=2 / 3$ where $\rho=N / L$ is the electron concentration, $N$ is the number of electrons 5. 7. In that cases the ground state is charge-ordered and insulating for small enough $t$, except special parameter ratios $\left(V=2 V^{\prime}\right.$ for $\rho=1 / 2$ and $V=0$ or $V^{\prime}=0$ for $\left.\rho=2 / 3\right)$ when the ground state has macroscopically large degeneracy $g=\exp (L S), S$ is the entropy per cite in the limit $t=0$. Consider now a case $1 / 2<\rho<2 / 3$. It is easy to demonstrate that $S$ is finite at $t=0$ and arbitrary positive $V$ and $V^{\prime}$. Therefore one can expect that the ground state is gapless (conducting) at any small but non-zero $t$. However, the character of the electron motion turns out to be different for $V>2 V^{\prime}$ and $V<2 V^{\prime}$. In the former case the processes of the motion of a single electron without the change of the energy of Coulomb repulsion are possible but in the latter one the electrons can move only as a coupled pairs on neighbouring sites (bipolarons). For example, the configurations [...011011001...] and [..011001101...] which are distinguished by the motion of one bipolaron in right direction have the same interaction energy (here 1 and 0 are labels for occupied and empty sites). At the same time, the configurations $[\ldots 010111001 \ldots]$ or $[\ldots 011010101 \ldots]$ have the additional energy equal to $V^{\prime}$ and $2 V^{\prime}-V$, correspondingly. The latter is the value of bipolaron binding energy at $t=0, V^{\prime}<V<2 V^{\prime}$. Note that this bipolaron state is similar to Shubin-Vonsovsky "maximum polarity" state 11. Thus, the cases $V>2 V^{\prime}$ and $V<2 V^{\prime}$ are physically different and has to be considered separately. We investigate both of them numerically for the case $\rho=4 / 7$. Parameters of the ground state, correlation functions, etc. are calculated by the Lanczos method with the extrapolation to the infinite length of the chain $L \rightarrow \infty$; the computational methods are described in Refs. [5, 7].

Consider first the region $V>2 V^{\prime}$. One can assume that the system is Luttinger liquid (LL) 12] in this case. There are three branches of low-energy excitations in LL: density fluctuation, current, and charge excitations with the velocities $v_{S}, v_{J}$ and $v_{N}$, correspondingly. The first one is connected with the variation of the total energy $E$ of the system under the variation of the total momentum $P, v_{S}=\delta E / \delta P$; the second one $\left(v_{J}\right)$, with the variation of the energy under a shift of all the particles in momenta space, and the third one, with the variation of the chemical potential $\mu$ under the change of the total number of particles $N, v_{N}=(L / \pi) \delta \mu / \delta N$. The velocity $v_{J}$ is connected with the conducting properties of the system [13, 14]: $v_{J}=2 \pi D$ where $D$ is the Drude weight (a spectral weight of the zero-frequency peak in the conductivity). Thus $v_{J} \neq 0$ is one of the criterion of the metallic state 13 . As it was shown by Haldane 12], the identity

$$
\left(v_{S}\right)^{2}=v_{J} v_{N}
$$

takes place. Therefore it is possible to introduce the parameter

$$
e^{-2 \varphi}=v_{N} / v_{S}=v_{S} / v_{J}
$$


which determines the time and space asymptotics of the correlation functions of the system. One-electron distribution function $n(k)$ as a function of the quasimomentum $k$ has a power-low singularity near the Fermi surface, $k=k_{F}$

$$
n(k) \approx n\left(k_{F}\right)-C \operatorname{sgn}\left(k-k_{F}\right)\left|k-k_{F}\right|^{\alpha} .
$$

with $\alpha=\cosh 2 \varphi-1$. One-particle local spectral density $\rho(E)$ has a singularity near the Fermi energy $\mu$ with the same exponent, $\rho(E) \propto|E-\mu|^{\alpha}$ which can be measured therefore by photoemission spectroscopy. There are various experimental data for inorganic materials with charge-density-waves 15, 16, 17] as well as for the organic superconductor (TMTSF) ${ }_{2} \mathrm{PF}_{6}$ [18] which confirms power-law singularity in $\rho(E)$ predicted by LL theory. It is interesting that in the latter case the value $\alpha \simeq 1.25$ has been found. At the same time, in all the known models with short-range interactions we always have $\alpha<1$ (in particular, in the 1D Hubbard model one can prove that $\alpha \leq 1 / 8[19])$. Our numerical calculations show that $\alpha<1$ is also in the model under consideration for one-half or two-third- fillings [7]. Here we demonstrate that $\alpha$ can be larger that 1 for $1 / 2<\rho<2 / 3$ which give a qualitative explanation of the experimental data 18.

The computational results are presented in the Table.

Table 1: Velocities $v_{J}, v_{N}$, and $v_{S}$ extrapolated to $L \rightarrow \infty$, the ratio $\chi=$ $v_{J} v_{N} /\left(v_{S}\right)^{2}$, and the parameter $\alpha$ from Eq. (伭) for different $V, V^{\prime}(t=1)$. For the lower part of the Table there is no Luttinger liquid state $\left(\chi, v_{N} \rightarrow \infty\right.$ at $L \rightarrow \infty$ and $\alpha$ is not defined).

\begin{tabular}{|c|c|c|c|c|c|c|}
\hline$V$ & $V^{\prime}$ & $v_{J}$ & $v_{N}$ & $v_{S}$ & $\chi$ & $\alpha$ \\
\hline 2 & 0 & 1.798 & 4.747 & 2.924 & 0.998 & 0.120 \\
2 & 0.5 & 1.877 & 4.591 & 2.947 & 0.992 & 0.101 \\
2 & 1 & 1.907 & 4.396 & 2.920 & 0.983 & 0.088 \\
2 & 1.5 & 1.889 & 4.328 & 2.892 & 0.978 & 0.088 \\
10 & 0 & 1.074 & 8.059 & 2.939 & 1.002 & 0.572 \\
10 & 5 & 1.668 & 9.314 & 3.806 & 1.065 & 0.408 \\
40 & 11 & 0.886 & 19.582 & 4.197 & 0.985 & 1.456 \\
80 & 22 & 0.816 & 20.848 & 4.132 & 0.997 & 1.624 \\
120 & 33 & 0.793 & 21.301 & 4.108 & 1.001 & 1.686 \\
\hline 10 & 10 & 0.269 & & 0.757 & & \\
10 & 15 & 0.124 & & 0.379 & & \\
10 & 20 & 0.070 & & 0.248 & & \\
10 & 40 & 0.030 & & 0.103 & & \\
10 & 60 & 0.019 & & 0.065 & & \\
20 & 20 & 0.104 & & 0.377 & & \\
40 & 40 & 0.052 & & 0.182 & & \\
80 & 80 & 0.026 & & 0.089 & & \\
100 & 60 & 0.107 & & 0.367 & & \\
\hline
\end{tabular}

The finiteness of $v_{J}$ and, consequently, of the Drude weight proves the metallic nature of the ground state. One can see that for $V>2 V^{\prime}$ Eq.(2) is valid (but 
not for $V<2 V^{\prime}$ where the system is not LL but bipolaron liquid, see below). The exponent $\alpha$ appears to be maximum along the line $V^{\prime}=(11 / 40) V$. In particular, for $V=120, V^{\prime}=33$ one has $\alpha \simeq 1.68$ which is even larger than the experimental value for $(\mathrm{TMTSF})_{2} \mathrm{PF}_{6}[18]$.

Consider now the case $V<2 V^{\prime}$. It is shown from the Table that Eq.(2) is violated in this case, at least, for large enough $V$ and $V^{\prime}$. As it was discussed above the reason is the formation of bipolarons. There are two evidences of the coupling of electrons in the pairs for large enough $2 V^{\prime}-V$. First of all, the energy gap defined as $\Delta=E(N+1)+E(N-1)-2 E(N)$ is finite (Fig. 1);

Figure 1: Energy gap as a function of $V^{\prime}$ at $V=20$ for $L=14(\circ), 21(\square), 28(\triangle)$, and the results of the extrapolation to $L \rightarrow \infty(\bullet)$.

at the same time, the Drude weight is also non-zero which means that $\Delta$ is the superconducting, or bipolaron, gap but not insulating one. Then, the ground state energy appears to be oscillate as a function of magnetic flux in the ring 
$\Phi$ with the period corresponding to the charge $2 e$ of current carriers. The corresponding results are presented in Fig. 2,

Figure 2: Flux dependence of the total energy of the ring $(L=21)$ for $V=20$ and different $V^{\prime}: V^{\prime}=12$ (diminished by a factor 2$)(\circ) ; V^{\prime}=14(\square) ; V^{\prime}=15(\triangle)$; $V^{\prime}=20(\nabla)$.

the flux being introduced by the replacement $t \rightarrow t \exp (2 \pi i \Phi)$ in Eq.(11). According to these criteria, bipolarons do not form at small enough $2 V^{\prime}-V \simeq$ $t$. To understand better this transition region we fit the total energy by the expression

$$
E(\Phi)-E(0)=\beta(1-\cos 2 \pi \Phi)+\gamma(1-\cos 4 \pi \Phi)
$$

where the first term corresponds to the "normal" current carriers and the second one to the bipolarons. The fitted parameters $\beta$ and $\gamma$ are shown in Fig. 3.

One can see that there is a coexistence of bipolaron conductivity and the usual one in the transition region $V \simeq 2 V^{\prime} \gg t$. It would be very interesting to 
Figure 3: Fitted parameters $\beta / \sqrt{\beta^{2}+\gamma^{2}}(\bullet)$ and $\gamma / \sqrt{\beta^{2}+\gamma^{2}}$ (o) from Eq.(5) as a function of $V^{\prime}$ for $V=20$ and $L=21$. 
investigate an explicit nature of this coexistence (phase separation or a homogeneous state) but for this aim computations for larger clusters are necessary.

In the limit of small $t$ one can simplify the problem using the operator perturbation theory developed first by Bogoliubov 20. In the lowest order in $t /\left(2 V^{\prime}-V\right)$ one can obtain

$$
H_{\text {eff }}=-\frac{t^{2}}{2 V^{\prime}-V} \mathcal{P} \sum_{i=1}^{L}\left(c_{i}^{\dagger} c_{i+1} c_{i-1}^{\dagger} c_{i}+c_{i}^{\dagger} c_{i-1} c_{i+1}^{\dagger} c_{i}\right) \mathcal{P} .
$$

where $\mathcal{P}$ is the projection operator on the state with the lowest Coulomb energy. This Hamiltonian describes the bipolaron motion with the effective transfer integral $t^{2} /\left(2 V^{\prime}-V\right)$. Our numerical results give for the Drude weight

$$
v_{J}=2 \pi D \simeq \frac{2.3 t^{2}}{2 V^{\prime}-V}
$$

For small enough $t$ the latter can be arbitrarily small. This differ drastically the case under consideration from the usual Verwey transition. One can show for $\rho=1 / 2, V^{\prime}=0$ that near the transition point $V=2 t$ the Drude weight has a finite jump 14

$$
v_{J}(V=2 t-0)=\pi / 2 ; \quad v_{J}(V=2 t+0)=0 .
$$

To conclude, we investigate numerically the ground state of 1D spinless fermion model with competing interactions beyond half-filling. Depending on the ratio of NN and NNN interactions it is either Luttinger liquid (with unusually large exponent $\alpha$ which can be even larger than 1) or bipolaron liquid. These results may be important to understand properties of low-dimensional conductors; in particular, the value $\alpha>1$ has been observed in photoemission experiments for (TMTSF) ${ }_{2} \mathrm{PF}_{6} 18$.

This work is partially supported by Russian Basic Research Foundation, grant 00-15-96544.

\section{References}

[1] P. Fazekas and P. W. Anderson, Philos. Mag. 30, 423 (1974)

[2] P. W. Anderson, The Theory of Superconductivity in the High-T Cuprates(Princeton Univ. Press, Princeton, 1997)

[3] V. Yu. Irkhin and M. I. Katsnelson, Pis'ma ZhETF 49, 500 (1989) [JETP Lett. 49, 576 (1989)]; Phys. Lett. A 150, 47 (1990)

[4] B. Canals and C. Lacroix, Phys. Rev. B 61, 1149 (2000)

[5] A. K. Zhuravlev, M. I. Katsnelson, and A. V. Trefilov, Phys. Rev. B 56, 12939 (1997) 
[6] E. V. Tsiper and A. L. Efros, J. Phys.: Condens. Matter 9, L561 (1997)

[7] A. K. Zhuravlev and M. I. Katsnelson, Phys. Rev. B 61, 15534 (2000)

[8] E. J. W. Verwey and P. W. Haaymann, Physica 8, 979 (1941)

[9] C. Schlenker and M. Marezio, Philos. Mag. B 42, 453 (1980); B. K. Chakraverty, ibid., 42, 473 (1980)

[10] K. Kobayashi, T. Susaki, A. Fujimori, T.Tonogai, and H.Takagi, condmat/9909189

[11] S. P. Shubin and S. V. Vonsovsky, Phys. Z. Sowjetunion 7, 292 (1935); 10, 348 (1936); S. V. Vonsovsky and M. I. Katsnelson, J. Phys. C 12, 2043 (1979)

[12] F.D.M. Haldane, Phys. Rev. Lett. 47, 1840 (1981); J. Phys. C 14, 2585 (1981)

[13] W. Kohn, Phys. Rev. 133, A171 (1964)

[14] B. S. Shastry and B. Sutherland, Phys. Rev. Lett. 65, 243 (1990)

[15] B. Dardel, D. Malterre, M. Grioni, P. Weibel, Y. Baer, and Levy F., Phys. Rev. Lett. 67, 3144 (1991)

[16] B. Dardel, D. Malterre, M. Grioni, P. Weibel, Y. Baer, C. Schlenker, and Y. Petroff, Europhys. Lett. 19, 525 (1992)

[17] Y. Hwu, P. Almeras, M. Marsi, H. Berger, F. Levy., M. Grioni, D. Malterre, and G. Margaritondo, Phys.Rev. B 46, 13624 (1992)

[18] B. Dardel, D. Malterre, M. Grioni, P. Weibel, Y. Baer, J. Voit, and D. Jerome, Europhys. Lett. 24, 687 (1993)

[19] H. J. Schulz, Phys. Rev. Lett. 64, 2831 (1990); M. Ogata and H. Shiba, Phys. Rev. B 41, 2326 (1990); H. Frahm and V. E. Korepin, Phys. Rev. B 43, 5653 (1991)

[20] N. N. Bogoliubov, Lectures in Quantum Statistics (Radianska Shkola, Kiev, 1949) 


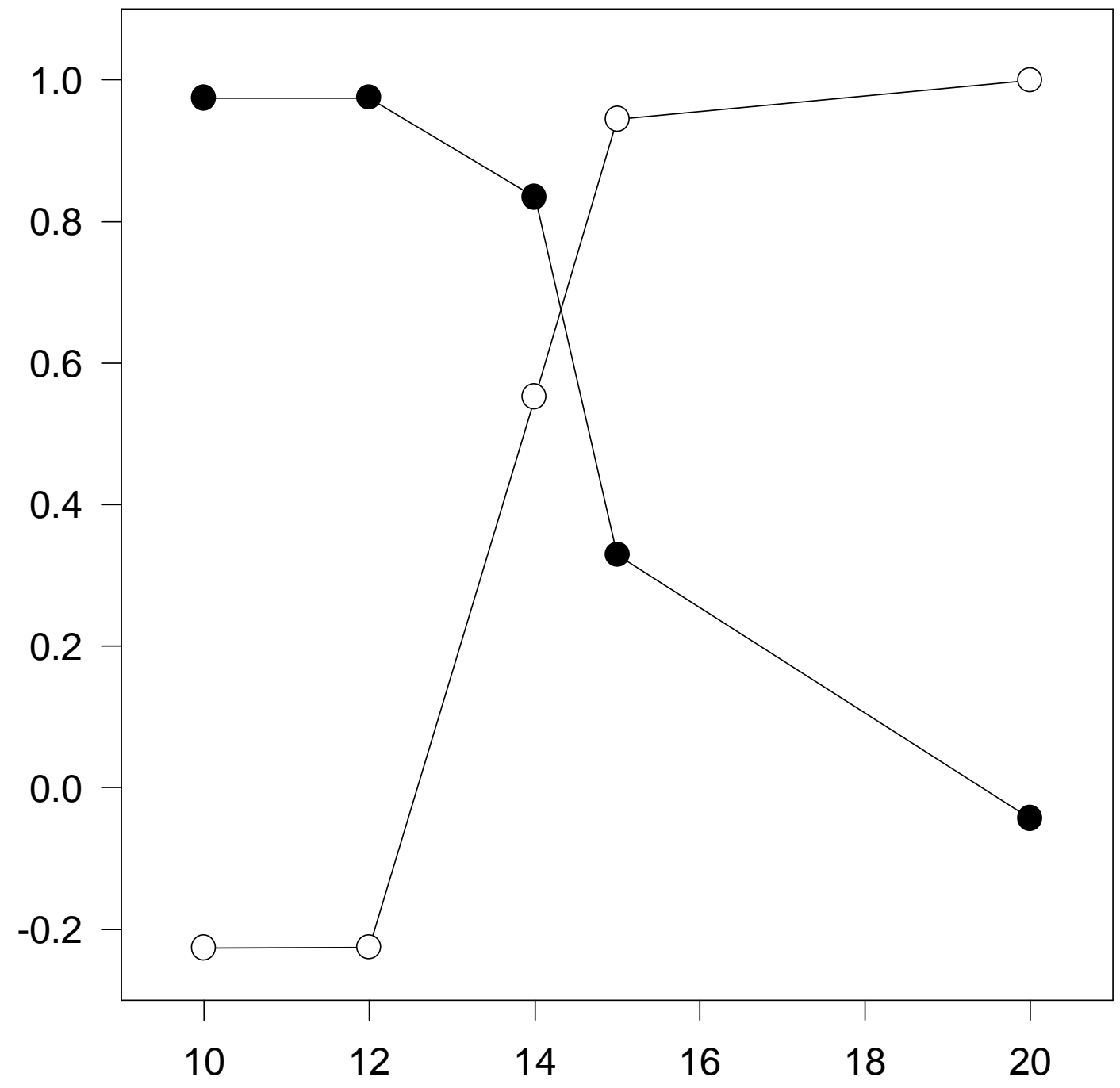

$V^{\prime}$ 


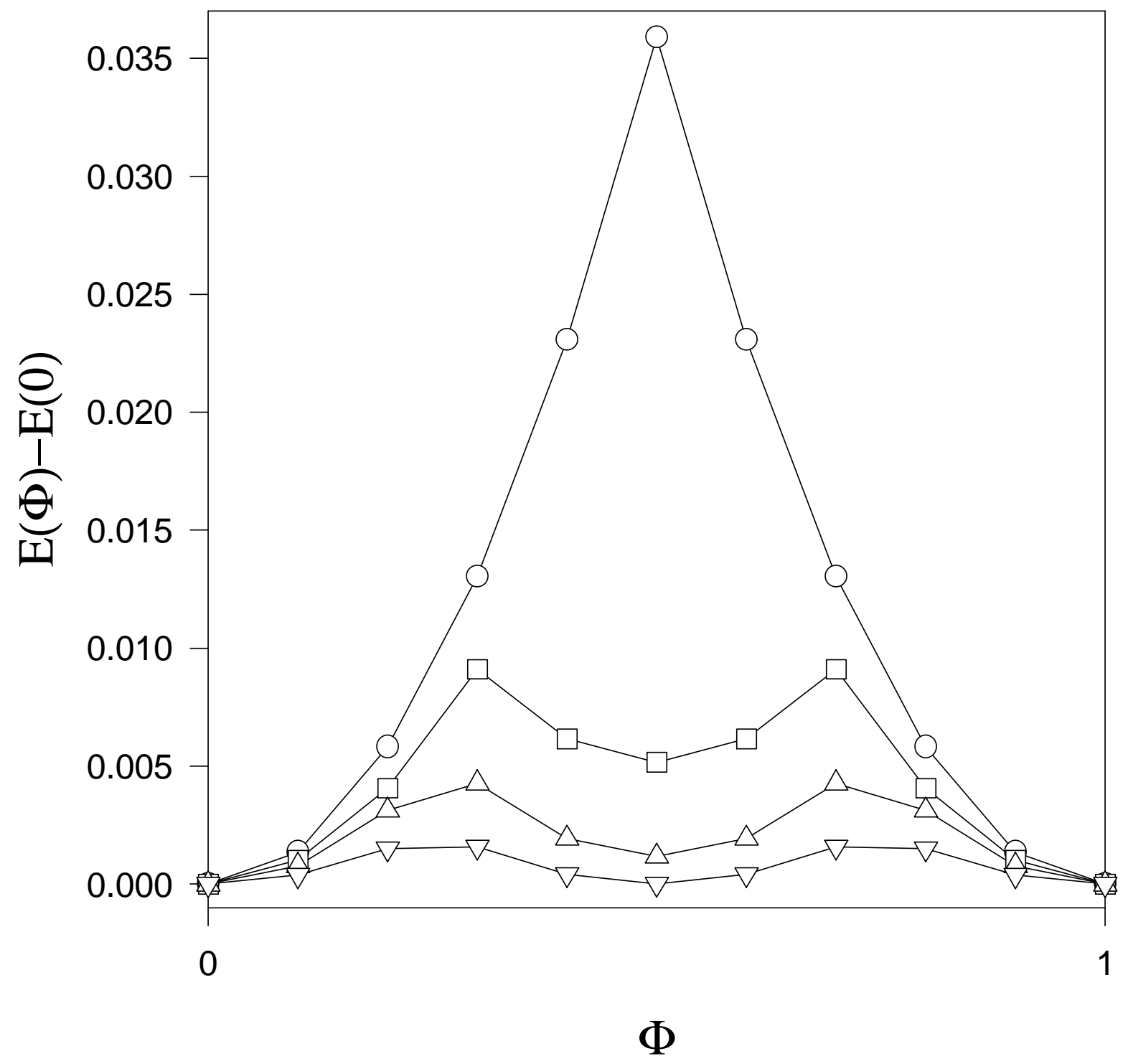


$\Delta$

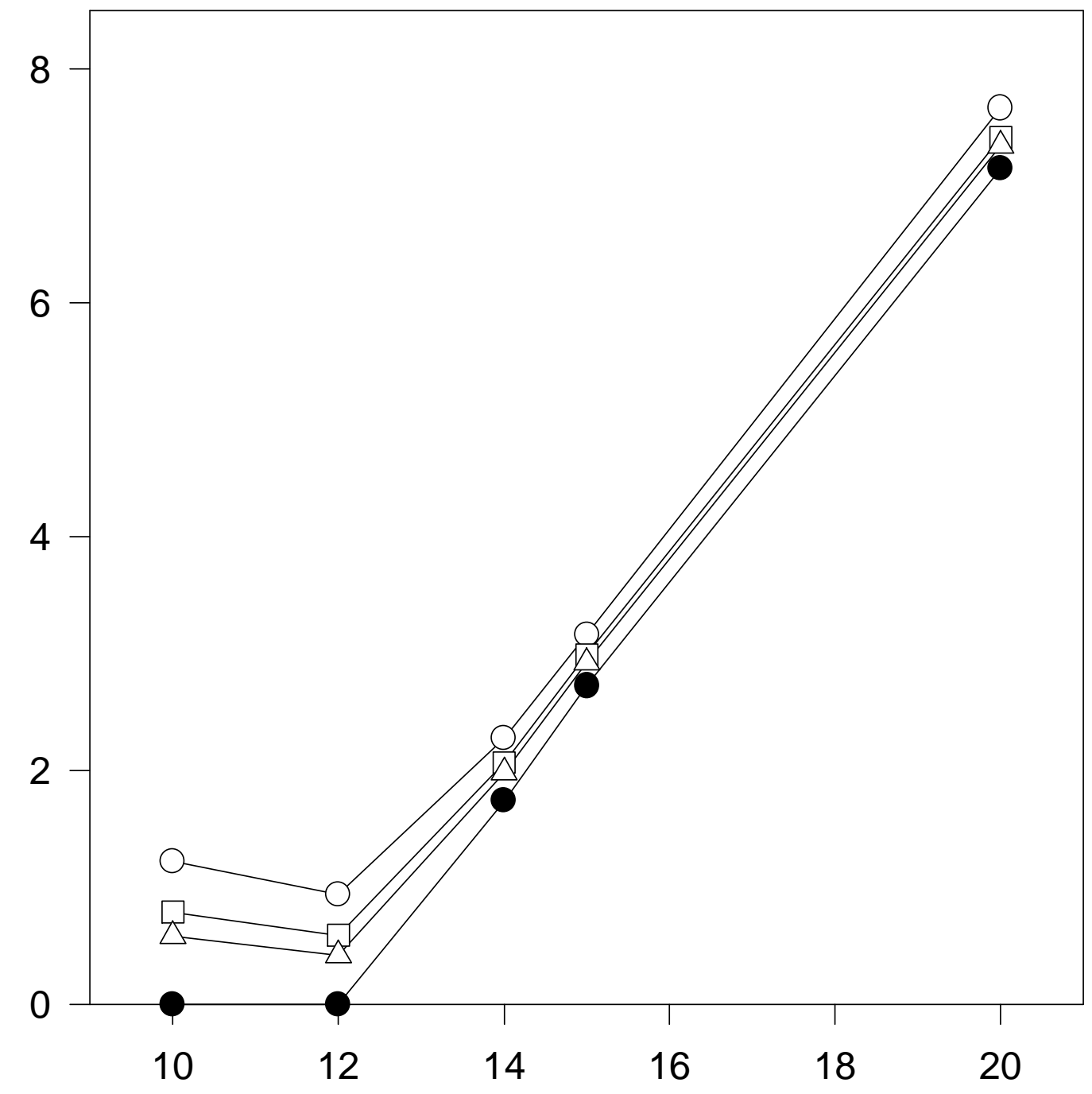

